\title{
Predicted RNA secondary structures for the conserved regions in dengue virus
}

\author{
Pallavi Somvanshi*, Prahlad Kishore Seth \\ Bioinformatics Centre, Biotech Park, Sector G, Jankipuram, Lucknow 226021, Uttar Pradesh, India \\ Pallavi Somvanshi - E-mail: psomvanshi@gmail.com; Tel.: +91 522 4012076; fax: +91 522 4012081; *Corresponding author \\ Received December 03, 2008; revised April 03 2009; accepted April 16, 2009; published August 2, 2009
}

\begin{abstract}
Dengue fever, dengue hemorrhagic fever and dengue shock syndrome are the prevalent mosquito borne viral infections worldwide. The dengue virus belongs to the genus flavivirus with conserved RNA domains peptidase_S7 and dexHc among its members. The secondary structures for RNA domains peptidase_S7 and DexHc are hence predicted and discussed with other known viral RNA structures to glean structural insights through comparison.
\end{abstract}

Keywords: RNA, dengue; structure; prediction; thermodynamics; pathogenicity

\section{Background:}

Dengue viral infection poses a growing public health problem in various tropical and subtropical countries. Dengue was circulated as a quasi-species, which is categorized into four serotypes [1]. Dengue virus belongs to the genus Flavivirus of the family Flaviviridae [2]. There are four serotypes of dengue virus (DEVI-IV) which causes dengue hemorrhagic fever and dengue shock syndrome [2, 3]. The infection caused by dengue viruses are widely recognized as a major public health concern, with more than one million cases of dengue hemorrhagic fever (DHF) per year with fatality rates 1 to $10 \%$. The most susceptible to the disease are children and young adults.

Dengue is a positive stranded RNA virus and the genome encodes a single polyprotein [4]. A comprehensive assessment of phylogenetic relationship of genus Flavivirus was determined. Three RNA domains (Peptidase_S7, DEXHc and Flavi_NS5) were found conserved in the genus Flavivirus. The conserved RNA domains help in processing of the polyprotein into mature protein subunits. DEXHc contributes in unwinding of nucleic acids for various aspects of RNA metabolism, nuclear transcription, pre mRNA splicing, ribosome biogenesis, nucleo-cytoplasmic transport, translation, RNA decay and organellar gene expression and flavi_NS5 that possess a number of short regions and motifs homologous to other RNA directed RNA polymerases [5].

The development of an anti-viral drug or vaccine target is still a non-trivial task for dengue virus. Therefore, it is important to understand RNA structures. RNA structures are characterized with secondary structures and are less complex than protein structures. It is also known that the single stranded RNA structures are often stabilized hydrogen bonds [6]. MFold is a well known tool used for prediction of RNA secondary structure. RNA secondary structure plays an important role viral multiplication. It is known that the evolution of virus RNA genome is subjected to various structural constrains including RNA structures [7]. Therefore, it is important to predict and discuss RNA secondary structures for conserved domains of peptidase_S7 and DexHc in dengue virus.

\section{Methodology:}

Dengue genome sequence and conserved domain

The complete genome sequence of dengue viruses was downloaded from GenBank at National Center for Biotechnological Information (NCBI) [8] and the Universal Virus Database of the International Committee on Taxonomy of Viruses genome database (ICTVdB) [9]. The complete DNA sequences of dengue virus were used to identify the conserved domain using the conserved domain database (CDD) at NCBI.

RNA secondary structure prediction package Prediction of RNA folding of the conserved domains of dengue virus was completed using the online MFold package [10]. MFold is the most widely used algorithms (uses genetic algorithm) for RNA secondary structure prediction, which are based on a search for the minimal free energy state. The algorithm allows structures to be removed at later stages of the simulation if other pairings are found to be more favorable. This is in addition to the possibility of growing of new stems. The algorithm also allows the prediction of certain tertiary interactions (example, RNA pseudo-knots).

\section{Discussion:}

The RNA genome of dengue virus contains conserved domains of peptidase_S7 and dexHc among different strains (Table 1 in supplementary material). Therefore, it is important to predict their secondary structures (Figure 1 and Figure 2). We used MFold (software package) to predict their secondary structures with energy calculations (Table 2). The dengue I stain showed the highest free energy for peptidase_S7 with $\delta \mathrm{G}-38.50$ and DexH with $\delta \mathrm{G}-30.50$. However, the dengue IV virus showed the lowest free energy for peptidase_S7 (with $\delta \mathrm{G}-42.30$ ) and DexHc (with $\delta \mathrm{G}$ 42.80). We find structural similarity with other predicted RNA structures described henceforth below. 


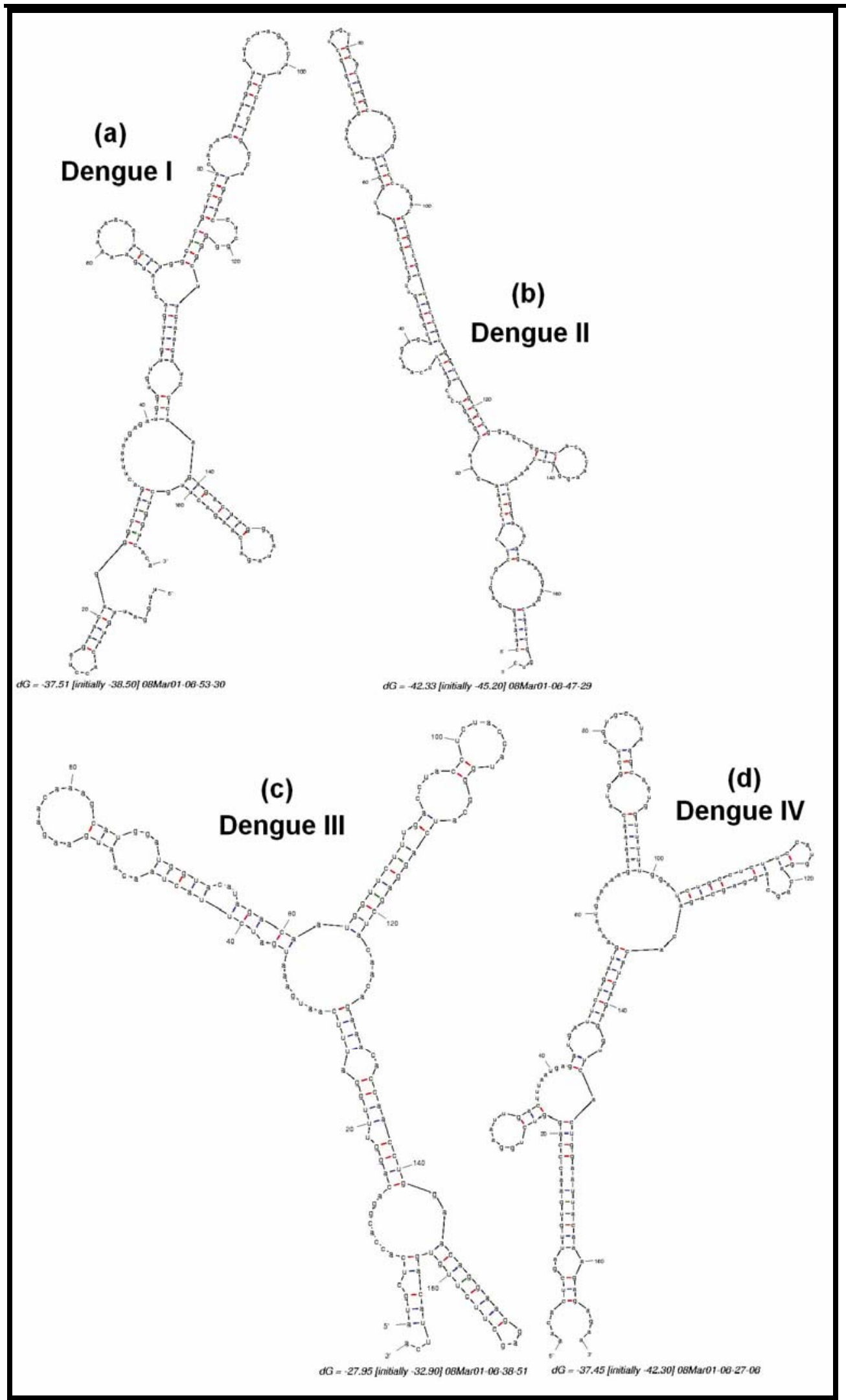

Figure 1: RNA secondary structures of peptidase_S7 conserved domain for dengue serotypes. 


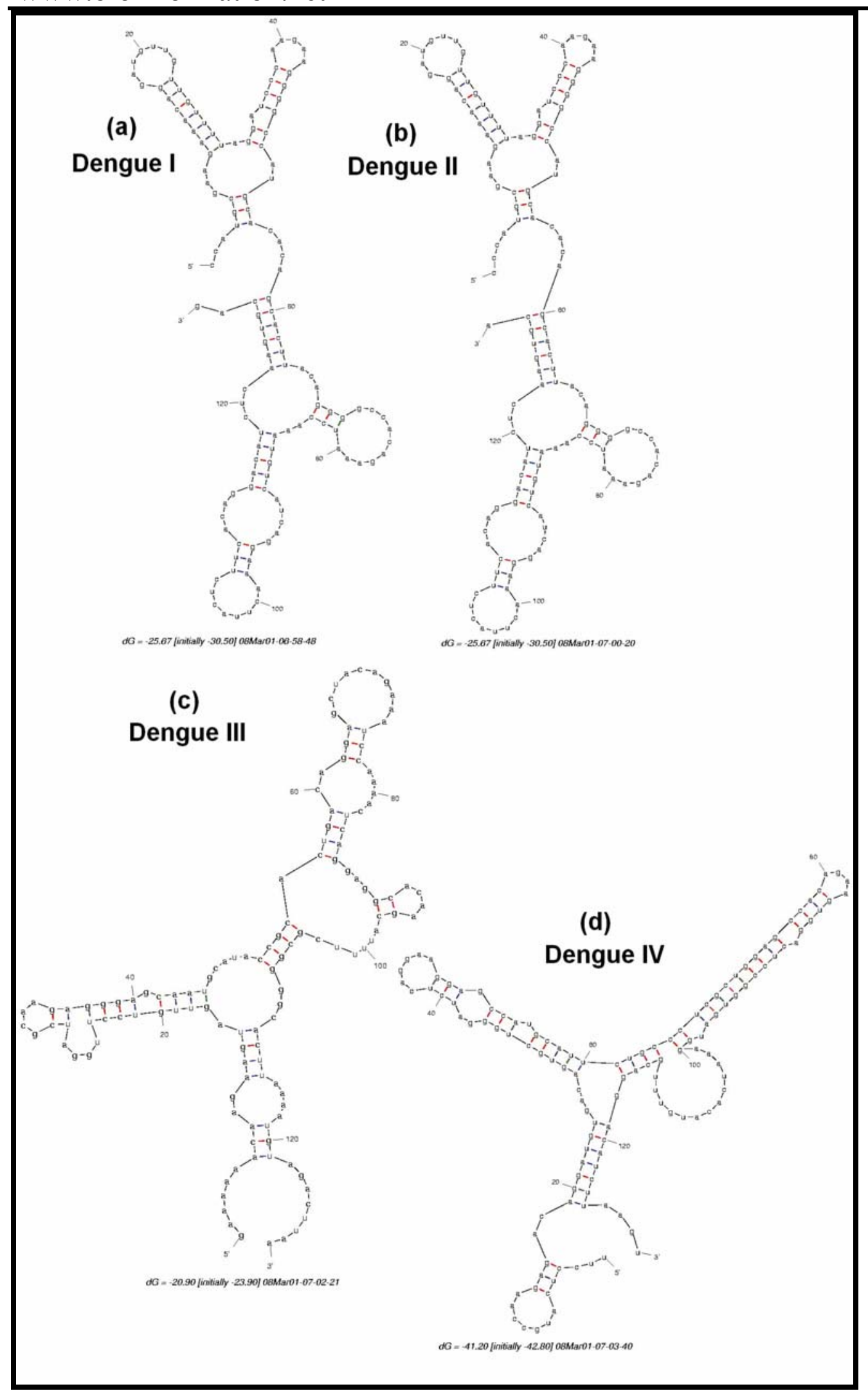

Figure 2: RNA secondary structures of DeXHc conserved domains of dengue serotypes. 


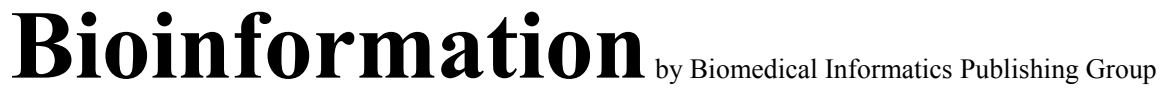

WWw.bioinformation.net

Several reports are available on the RNA secondary structure prediction. The genomic diversity of argentine tospo viruses from different geographical areas with several distinct crops has been reported. A $450 \mathrm{nt}$ fragment of the $\mathrm{N}$ gene were substantially described. A partial sequence of the $\mathrm{N}$ gene was able to classify local isolates within three tospo virus species previously described (Tomato spotted wilt virus, TSWV; tomato chlorotic spot virus, TCSV and groundnut ring spot virus, GRSV) [11]. Six evolutionarily conserved stem-loop structures in the NS5B encoding region and two in core gene have been reported. This observation relates to that found in $\mathrm{HCV}, \mathrm{GB}$ virus-B (GBV-B) with similar internal base pairing in its coding region [12].

Hepatitis $\mathrm{C}$ virus (HCV) possesses extensive RNA secondary structure in the core and NS5B-encoding regions of the genome. A program (STRUCTUR_DIST) was developed to the analyses of multiple RNA folding patterns predicted using MFOLD to determine the evolutionary conservation of predicted stem loop structures. This method helps to analyse frequencies of covariant sites in predicted RNA folding between HCV genotypes [13]. A comparison of the structure of the 3 '-untranslated region ( $3^{\prime}-\mathrm{UTR}$ ) of $\mathrm{HGV} / \mathrm{GBV}-\mathrm{C}$ with the upstream NS5B coding sequence has been shown. The secondary structure predictive algorithms and analysis of covariance between $\mathrm{HGV} / \mathrm{GBVC}$ genotypes $1-4$ and the more distantly related $\mathrm{HGV} / \mathrm{GBV}-\mathrm{C}$ chimpanzee variant, show RNA secondary structure formation in both regions. In particular, the NS5B region contained long stem-loop structures of up to 38 internally paired nucleotides which were evolutionarily conserved between human, chimpanzee and HGV/GBV-C variants [14]. The NS1 gene of Influenza virus is stable during evolution. The computational tool has been used to model its RNA secondary structure (free energy ranged between -222.90 to- $251.10 \mathrm{Kcal} / \mathrm{mol}$ ) for nine different strains of Influenza A virus [15].

RNA secondary structure prediction was combined with comparative sequence analysis to construct models of folding for the distal 380 nucleotides of the 3'-untranslated region (3'-UTR) of Yellow fever virus (YFV). A number of structural elements that are thermodynamically stable, conserved in shape, and confirmed by compensatory mutations have been shown [16]. At the same time structural polymorphisms were observed among strains of YFV. The observation of a strong association between secondary structure of the 3 -UTR and virulence of YFV may help elucidate the molecular mechanisms of virus attenuation. This may lead to the development of new strategies directed towards rational modification of secondary structure of the 3`-UTR.

Prediction of evolutionarily conserved secondary structure motifs in the genomic RNAs of the family Flaviviridae has been reported [17]. This virus family consists of the three genera Flavivirus, Pestivirus, Hepacivirus and a group of GB virus $\mathrm{C} /$ hepatitis $\mathrm{G}$ virus (with an uncertain taxonomic classification). RNA secondary structures for 5S rDNA of 37 bacteria have been investigated [18]. Data show that the lowest free energy of the $5 \mathrm{~S}$ rDNA is related to the most primitive bacteria and high free energy always indicates less stability during the evolution.

\section{Conclusion:}

The prediction of RNA secondary structure was performed for peptidase_S7 and DexHc of dengue virus serotypes. The predicted structures of conserved RNA domains provide structural insights for potential function in RNA mediated viral replication.

\section{Acknowledgement:}

We thank Indian Council of Medical Research, New Delhi (64/2/07/BIF-BMS) and Department of Biotechnology, New Delhi.

\section{Reference:}

[1] J. Mongkolsapaya et al., J Immunol., 176(6): 3821 (2006)

[2] D. J. Gubler Trends Microbiol., 10:100 (2002).

[3] S. B. Halstead Science 239:476 (1988).

[4] F. Billoir et al., J Gen. Virol., 81:781 (2000).

[5] http://www.ncbi.nlm.nih.gov/Structure/cdd/wrpsb.cgi

[6] E. Zamaratski et al., Nucleosides, Nucleotides \& Nucleic Acids 20: 1219 (2001)

[7] P. Simmonds et al., RNA 10:1337 (2004).

[8] http://www.ncbi.nlm.nih.gov/genomes/VIRUSES/viru ses.html

[9] http://www.ncbi.nlm.nih.gov/ICTVdb/

[10] M. Zuker Science 244:48 (1989).

[11] R.A. Dewey et al., Acta Horticulturae 431: (1996)

[12] A. Tuplin et al., RNA 8:824 (2002).

[13] A. Tuplin et al., J. Gen. Virol. 85:3037 (2004).

[14] N. M. Cuceanu., et al., J. Gen. Virol. 82:713 (2001).

[15] P. Somvanshi et al., J Proteomics Bioinform 1:219 (2008).

[16] V. Proutski et al., J. Gen. Virol. 78: 1543 (1997).

[17] A. Thurner et al., J. Gen. Virol. 85: 1113 (2004).

[18] V. Singh et al., J. mol. Graphics mod. 29:770(2009)

Edited by $P$. Kangueane

Citation: Somvanshi \& Seth, Bioinformation 3(10): 435-439 (2009)

License statement: This is an open-access article, which permits unrestricted use, distribution, and reproduction in any medium, for non-commercial purposes, provided the original author and source are credited. 


\section{Supplementary material}

Table 1: Conserved regions in RNA of dengue virus

\begin{tabular}{llcc}
\hline Virus Name & Accession No & Peptidase_S7 & DeXHc \\
\hline Dengue 1 & NC_001477 & $1482-1652$ & $1665-1794$ \\
Dengue 2 & NC_001474 & $1482-1652$ & $1664-1793$ \\
Dengue 3 & NC_001475 & $1480-1650$ & $1663-1792$ \\
Dengue 4 & NC_002640 & $1481-1648$ & $1663-1792$ \\
\hline
\end{tabular}

Table 2: Free energies $(\delta G)$ of different dengue virus strains

\begin{tabular}{clrr}
\hline S. No. & Strain/ conserved domain & base & $\begin{array}{c}\delta \mathrm{G} \text { value } \\
(\mathrm{Kcal} / \mathrm{mol})\end{array}$ \\
\hline 1 & DEV-I Peptidase_S7 & 171 & -38.50 \\
2 & DEV-II Peptidase_S7 & 170 & -38.10 \\
3 & DEV-III Peptidase_S7 & 170 & -32.90 \\
4 & DEV-IV Peptidase_S7 & 167 & -42.30 \\
5 & DEV-I DexHc & 129 & -30.50 \\
6 & DEV-II DexHc & 129 & -30.50 \\
7 & DEV-III DexHc & 129 & -23.90 \\
8 & DEV-IV DexHc & 129 & -42.80 \\
\hline
\end{tabular}

